\title{
Neue Targeted-Therapie bei fortgeschrittenem Nierenzellkrebs
}

- Der orale Angiogenese-Hemmer Pazopanib (Votrient ${ }^{\circledR}$ ) erweitert das Spektrum der zielgerichteten Therapien beim fortgeschrittenen Nierenzellkarzinom. Seine Wirksamkeit und Verträglichkeit wurde in einer multizentrischen placebokontrollierten Doppelblindstudie untersucht, an der 435 Patienten mit metastasiertem klarzelligen Nierenzellkarzinom teilnahmen. Sie waren entweder unbehandelt oder hatten bereits Zytokine erhalten, berichtete Prof. Gero Kramer, Wien.

Die Ergebnisse zeigen eine signifikante Verlängerung der progressionsfreien Überlebenszeit (PFS) im Vergleich zu Placebo. Der Effekt auf das PFS war unabhängig davon, ob die Patienten bereits mit Zytokinen vorbehandelt waren oder nicht.
In der Verumgruppe lag die Ansprechrate bei $30 \%$, in der Placebogruppe bei $3 \%$ (p $<0,001$ ).

Eine Subgruppenanalyse hat ergeben, dass der Effekt auf das PFS nicht von MotzerRisiko, Alter, Geschlecht und ECOG-Performance-Status der Patienten beeinflusst wurde. Eine Interimsanalyse deutet laut Kramer darauf hin, dass die Substanz auch einen günstigen Einfluss auf das Gesamtüberleben hat.

Typische Nebenwirkungen von Tyrosinkinasehemmern wie Hand-Fuß-Syndrom, Thrombozytopenie und Mukositis beziehungsweise Stomatitis traten in der Studie insgesamt bei jeweils weniger als $10 \%$ der Patienten auf, vom Schweregrad drei oder vier bei weniger als $1 \%$ der Patienten.
Nach den Worten von Prof. Manuela Schmidinger, Wien, ist Pazopanib wegen seines guten Sicherheitsprofils prädestiniert für die Erforschung neuer Kombinationsstrategien.

aam

Satellitensymposium „Das Nierenzellkarzinom: Zeit für neue Fakten - neue Erfahrungen - neue Strategien “ im Rahmen der gemeinsamen Tagung der Bayerischen Urologenvereinigung und der Österreichischen Gesellschaft für Urologie und Andrologie, München, 11. Juni 2010. Veranstalter: GlaxoSmithKline, München

\section{Urologische Sommerakademie - ein Forum für Innovationen}

— Über 200 Urologen diskutierten auf der dritten Münchner Urologischen Sommerakademie über Neuigkeiten in ihrem Fachgebiet. Die Veranstaltung hatten die urologischen Universitätskliniken Großhadern und des Klinikums rechts der Isar organisiert - in Kooperation mit dem Unternehmen Astellas Pharma. Ein besonderer Höhepunkt der Veranstaltung war das „Oncoforum Urology“. Hier präsentierte die Sommerakademie die Highlights wichtiger urologischer Tagungen.

Ein wichtiges Thema war dabei das Prostatakarzinom (PCa): Die Chemoprävention des PCa hat sich bisher in praxi nicht durchgesetzt. Zwar zeigte die REDUCE-Studie bei der Einnahme von Dutasterid über vier Jahre hinweg bei über 6.700 Männern mit hohem PCa-Risiko eine relative Risikoreduktion von rund $23 \%$. Allerdings bestand immer die Befürchtung, dass das Wachstum höhergradiger Tumoren gefördert oder eine Umgebung geschaffen wird, in der höhergradige Tumoren einen Wachstumsvorteil haben.

Neuigkeiten gab es auch zur Hormontherapie bei lokal fortgeschrittenem $\mathrm{PCa}$ : So zeigte sich, dass die Kombination aus Hormontherapie und einer Bestrahlung im Vergleich zur alleinigen Androgendeprivation das tumorspezifische Überleben verbessert und zudem gut verträglich ist.

Ein weiteres Thema war die überaktive Blase, bei deren Therapie Anticholinergika Mittel der ersten Wahl sind. Wirksamkeit und Verträglichkeit dieser neuen Substanzen sind gut belegt. Vorteile für die Compliance können in der Einmalgabe und der Möglichkeit der individuell anpassbaren Dosierung liegen. Versagt eine Therapie mit Anticholinergika kann eine Injektion mit Botulinumtoxin helfen.

Diskussionsstoff boten auch Kasuistiken, wie die eines 56-jährigen Patienten, der an imperativem Harndrang litt - zum Teil mit Urinverlust. Alpha-Blocker verbesserten die Obstruktion, nicht aber die Urge-Symptomatik. Erst unter Kombinationstherapie aus Alpha-Blocker und Solifenacin (Vesikur ${ }^{\circledR}$ ) kam es zu einer deutlichen Verbesserung der Drangsymptomatik. Die richtige Reihenfolge der verschiedenen Wirkstoffgruppen medikamentöser Therapie sowie eines operativen Eingriffs wurden anhand dieses Falles angeregt diskutiert.

\section{Nach Informationen von}

Astellas Pharma, München

\section{Budgetschonende OAB-Behandlung}

_ Seit April 2010 ist das zur Behandlung der überaktiven Blase (OAB) eingesetzte Medikament Trospiumchlorid (Spasmolyt ${ }^{\circledR}$ ) als N3-Packung mit 84 Tabletten, in den Wirkstärken $20 \mathrm{mg}$ und $30 \mathrm{mg}$ Trospiumchlorid, erhältlich. Ärzte können beide Wirkstärken budgetschonend verordnen, da der Preisvorteil umgerechnet auf die Tagestherapiekosten $20 \%$ beträgt. Die 30-mg-Wirkstärke hat zudem den Vorteil, dass sie in der 84er-Packungsgröße für Inkontinenzpatienten zuzahlungsfrei ist. Pro eingelöstes Rezept spart der Patient 6,03 EUR. Weitere Vorteile der neuen Packungsgröße sind die individuelle Dosisanpassung und die leichte Teilbarkeit der 30-mg-Tabletten. Außerdem bleibt die Therapiehoheit beim Arzt, da Patienten in der Apotheke genau das verordnete Präparat erhalten. Trospiumchlorid gehört zum Goldstandard bei der Therapie von OAB-Symptomen und verfügt über eine zuverlässige klinische Wirksamkeit, Sicherheit und Verträglichkeit.

Nach Informationen von Rottapharm|Madaus, Köln 